Revista Educación

ISSN: 0379-7082

ISSN: 2215-2644

revedu@gmail.com

Universidad de Costa Rica

Costa Rica

\title{
El complejo inicio de la práctica docente en contingencia del estudiantado novel en educación inclusiva en tiempos de COVID-19
}

Rangel Romero, Juan Carlos

El complejo inicio de la práctica docente en contingencia del estudiantado novel en educación inclusiva en tiempos de COVID-19

Revista Educación, vol. 46, núm. 1, 2022

Universidad de Costa Rica, Costa Rica

Disponible en: https://www.redalyc.org/articulo.oa?id=44068165013

DOI: https://doi.org/10.15517/revedu.v46i1.44760

\section{(c) (1) $\Theta$}

Esta obra está bajo una Licencia Creative Commons Atribución-NoComercial-SinDerivar 3.0 Internacional. 


\section{El complejo inicio de la práctica docente en contingencia del estudiantado novel en educación inclusiva en tiempos de COVID-19}

\author{
Juan Carlos Rangel Romero \\ Benemérita y Centenaria Escuela Normal del Estado de \\ San Luis Potosí, México \\ jrangel@beceneslp.edu.mx \\ iD https://orcid.org/0000-0002-1160-6724
}

\author{
DOI: https://doi.org/10.15517/revedu.v46i1.44760 \\ Redalyc: https://www.redalyc.org/articulo.oa? \\ id $=44068165013$
}

Recepción: 22 Diciembre 2020

Aprobación: 09 Marzo 2021

\section{Resumen:}

La práctica docente del profesorado falto de experiencia es el proceso de construcción de hechos en el contexto escolar, con el propósito de desarrollar competencias para la atención educativa. En tiempos de contingencia, iniciarla en aislamiento en casa dirige al estudiantado novel en educación inclusiva a llevarla a cabo en escenarios virtuales que permiten la construcción de vivencias que serán parte de su preparación profesional. El objetivo de este artículo es reflexionar sobre el complejo proceso en la formación de inicio, donde, a través de la investigación etnográfica, se logre conocer, desde los testimonios del estudiantado, la forma en la que recapacita la primera aproximación a la distancia durante el ciclo escolar 2020-2021. Este documento concluye en la idea de que las personas que estudian crean pensamientos acerca del ser profesional de la educación aún en encierro, así como un concepto de identidad en cuanto a ser docente en tiempos confusos.

Palabras Clave: Práctica docente, Experiencia profesional, Reflexión docente, Dificultad de aprendizaje, COVID-19, Educación inclusiva.

\section{Abstract:}

Teaching practices of less experienced teachers build on events within the school setting to help hone and develop better teaching skills. During contingencies, novice teachers have had to teach online due to the lockdown, thereby creating their own experience and initial training in an unconventional setting. The objective of this article is to reflect on this complex process. As a result of ethnographic research, student testimonies convey their perception about distance learning during the 2020-2021 school year. As a result, it is revealed that student teachers create thoughts about teaching under lockdown, thereby establishing the concept of their own teaching identity even during these uncertain times.

Keywords: Teaching Practice, Professional Experience, Teacher Reflection, Learning Difficulties, COVID-19, Inclusive Education.

\section{INTRODUCCIÓN}

El comienzo de la práctica profesional es aquella actividad en la que toda persona experta en desarrollo inicia el acercamiento a una realidad en la que comienza a experimentar la labor, para teorizar acerca del conocimiento y poner en juego saberes y destrezas que le den el acceso a adquirir competencias profesionales, -entiéndase estas como el conjunto de elementos asociados al éxito del desempeño de las personas (Escobar, 2005)-, que le permitan desarrollar habilidades propias para participar de manera correcta en las situaciones particulares de los diversos ámbitos de acción.

Para el profesorado implica, además, la construcción de saberes que respalden las acciones en la tarea escolar (Vergara, 2016). Para el profesorado novel, construir reflexiones acerca de las formas en las que se interrelacionan los conocimientos y las experiencias para construir una forma de ser docente, impacta en la generación y creación de la cátedra, las estrategias de enseñanza, la didáctica y el desarrollo de aprendizajes que sean eficientes en su futuro alumnado. 
La escuela formadora de maestros y maestras de San Luis Potosí, México (Benemérita y Centenaria Escuela Normal del Estado, BECENE), lleva a cabo este proceso al interior de escuelas de educación básica -desde la Secretaría de Educación Pública (SEP), se refiere a los niveles de preescolar, primaria y secundaria-, donde se inicia el acercamiento del estudiantado para aplicar teorías, generar ideas, proponer ajustes curriculares y experimentar su ser como docentes en contextos en los que desarrollarán la práctica profesional de manera laboral.

Bajo esta normalidad del proceso de formación del profesorado novel, comienza en el segundo año de la carrera en la licenciatura en educación inclusiva, que da inicio de forma natural a través de la práctica escolar que se desarrolla de la siguiente manera: el estudiantado accede a la escuela durante cinco horas siete días a la semana a lo largo de tres jornadas durante un total de quince días; está en el aula física, las clases presenciales y la interacción entre estudiantado y profesorado. Al regreso a la escuela normal, genera un sentido de profesión a través de la participación directa con su preparación formal en relación al plan de estudios de licenciatura. Se da continua reflexión con el resto de compañeros, compañeras y personas catedráticas acerca de las vivencias obtenidas a través del contacto en los contextos de experiencia y la práctica asume la naturaleza cultural del magisterio nacional, que, desde el Sindicato Nacional de los Trabajadores de la Educación (SNTE), se declara como la preparación en beneficio de la enseñanza de la niñez, con la frase "por una educación al servicio del pueblo" (SNTE, 2020).

Pero el 16 de marzo de 2020, por acuerdo número 02/03/20 ante la emergencia sanitaria por el síndrome respiratorio agudo severo por Coronavirus 2, que genera la enfermedad identificada como COVID-19 durante el ciclo escolar 2019-2020, se suspendieron las actividades en las escuelas de educación básica, media superior y superior en el territorio mexicano; esto fue declarado en el Diario Oficial de la Federación (DOF, 2020), por lo que se suspendidas las acciones escolares en el territorio nacional a partir del 23 de marzo de 2020. Este cambio tan rápido llevó a concluir el ciclo escolar de manera emergente en aislamiento, por medios de comunicación como el teléfono, la televisión y de forma virtual en algunos casos. Se convirtió de manera rápida en un modelo al que —el profesorado y el estudiantado- "no estábamos preparados para hacer frente" (Estudiante 1, comunicación personal, 23 octubre 2020).

Con la necesidad de continuar los programas de formación del ciclo escolar 2020-2021, aún en riesgo por COVID-19, la tarea escolar supone una nueva normalidad, la cual consiste en una capacidad de adaptación que conlleva a toda la comunidad (Rinesi et al., 2020), debido a que, con el transcurso de la contingencia, existe mayor información acerca de la enfermedad, y se concurre a vivencias que son posibles de retomar para seguir el desarrollo de los programas de estudio con un pensamiento de ajuste a la realidad. El continuar de la emergencia de salud y la formación de maestras y maestros plantea retos importantes para el desarrollo de las prácticas profesionales en el alumnado novel, en las que una nueva forma de enseñanza, aprendizaje, seguimientos, ejercicios, intervenciones y labores por parte de las personas discentes permita generar experiencias que den oportunidad de desarrollar reflexiones que se sumen al desarrollo del profesorado de nuevo ingreso al programa de acercamiento.

Para llevar esta acción que continúa a la distancia, a través de la investigación en etnografía educativa (Murillo y Martínez, 2010) y el uso de la estrategia discursiva (Ruíz et al., 2010), se dirige a la observación y reflexión de las prácticas culturales en las que el estudiantado novel de licenciatura en educación inclusiva, a través de sus testimonios, replantea ajustes al conocimiento y ayudantía del programa de iniciación a la práctica docente en los servicios de educación especial. Se asume la distancia, en aislamiento y en la construcción intrapersonal desde el hogar como nuevo escenario de experiencias, ahora con acercamientos de hasta cuarenta minutos de dos a tres veces por semana a través de plataformas de video llamada por internet.

La importancia de continuar con el acercamiento, aún en contingencia y desde casa, es permitir a la persona discente inexperta "constituir el primer acercamiento del estudiante al trabajo docente. A través de su inmersión como ayudante y participación en las actividades de enseñanza-aprendizaje (...), para documentar su experiencia y el trabajo que realiza el docente titular de educación especial” (SEP, 2018, p. 6). 
El nuevo escenario en contingencia - que ahora es el hogar- tiene como consecuencia la suspensión compuesta de las actividades laborales, donde la escuela física, promovía el contacto entre discentes, padres de familia estudiantado y profesorado, como parte de ese acercamiento al futuro trabajo.

Entonces, surgen preguntas de reflexión como: ¿de qué manera, las personas discentes normalistas noveles vivirán el acercamiento a la práctica?; ¿cómo llevarán a cabo la reflexión de ser maestros y maestras en formación? Este es un proceso nuevo que involucra creatividad, autoaprendizaje, formas de relación interpersonal y de interacciones que carecerán de ser desarrolladas a través del acercamiento físico en la formación inicial del grupo licenciado en educación inclusiva en tiempos COVID-19.

En atención a lo anterior, el artículo tiene tres objetivos específicos. El primero es identificar la importancia de la práctica en el acercamiento a las experiencias en la profesión desde el programa de estudios; el segundo es reflexionar acerca del pensamiento que desarrolla el estudiantado novel en los escenarios a distancia, donde la persona discente se acerca a situaciones de dificultad que se presentan en las prácticas y las concepciones que tiene en ellas; el tercero es ilustrar las nuevas experiencias educativas desde los testimonios del estudiantado en construcción de la experiencia, lo refleja la construcción de una identidad docente en edificación, aún en el hecho de vivir una realidad diferente entre profesorado y estudiantado (Arriaga, 2015).

El documento concluye con la idea de que, aún a la distancia, el estudiantado de licenciatura novel construye reflexiones que aportan valor para la construcción de su identidad como profesional de la educación y que serán, en otro momento, parte activa de su desempeño laboral.

\section{Desarrollo del tema}

\subsection{La importancia de la práctica docente en el estudiantado novel de educación inclusiva}

La práctica docente, para el profesorado en formación, constituye una compleja red de relaciones sociales y actividades, por medio de las cuales se aproximan a los escenarios reales de trabajo para teorizar sobre el aprendizaje y la enseñanza en su área de estudio acerca de lo que será su trabajo de vida, al permitirle desarrollar un principio de identidad con la profesión.

Entonces, la práctica docente es la actividad que se desarrolla dentro de un escenario escolar, en el que el profesorado novel en formación tiene ese primer acercamiento a la interacción social, presentación y enfrentamiento a situaciones, eventos y personas, y en el que se genera un sinfín de sentidos y significados (Téllez et al., 2014). Desde el plan y programa de estudio 2018 para la formación de personas licenciadas en educación inclusiva, esta acción está dirigida a "buscar una intención, la que es, la de reproducir, crear formas de enseñanza, aprendizaje, evaluación, comunicación y trabajo en lo que se identificará como, cultura escolar" (SEP, 2018, p. 5).

La reflexión acerca de la función docente permite que el estudiantado novel de la escuela normal brinde, desde el acercamiento inicial, un sentido a la educación, y comience así la construcción de su ser como maestra o maestro. A través del análisis en el aula y la vivencia presencial en los campos educativos, la práctica le invita a construir y fortalecer un cúmulo de habilidades, destrezas, conocimientos y actitudes señaladas como competencias docentes, las que corresponden a "la movilización de recursos intelectuales profesionales para utilizar determinados objetos de aprendizaje, mediante metodologías didácticas pertinentes con profesionalismo ético en un contexto definido" (Castro, 2015 p. 265).

Dentro de las competencias específicas en el programa de estudios vigente de 2018 para la licenciatura en educación inclusiva, se plantea que el estudiantado será capaz de identificar y atender "las necesidades educativas del estudiantado con discapacidad, incorporar los recursos idóneos para el favorecimiento de aprendizajes, el apoyo a los docentes en las escuelas en el diseño y la planificación de actividades para la inclusión educativa" (SEP, 2018, p.9), por lo que la interacción, socialización, comunicación humana y 
contacto asumen desde la prácticas una importante actividad en el desarrollo de las acciones del programa educativo del profesorado, que le oriente a dar respuesta a esta condición propia de su actuar profesional.

\subsection{El inicio a la práctica docente en personas licenciadas en educación inclusiva. La primera práctica en contingencia del profesorado novel}

Llevar a cabo el análisis del trayecto experiencial del estudiantado en educación inclusiva es importante para entender, desde la mirada de quienes estudian, la manera en la que inicia la práctica docente, alejada del programa en la interacción física, pero en acercamiento a la búsqueda de la experiencia en contingencia. Este tipo de estudio es una actividad dinámica, reflexiva y que comprende los acontecimientos ocurridos en la interacción del profesorado y las personas en proceso de formación (García et al., 2008).

Desde la reflexión docente novel, el comienzo de las actividades de acercamiento a la práctica da inicio formal con el contacto con las autoridades educativas, lo que ahora implica llevar los procesos tradicionales de manera virtual con el profesorado y estudiantado para comenzar con el objetivo del programa, que es el contacto con los servicios de educación especial, los cuales inician en el mes de septiembre de 2020. A partir de esta organización, la asignación del estudiantado con docentes dirige a la participación en escuelas de educación básica que cuenten con Unidad de Servicios y Apoyos a la Educación Regular (USAER):

Este es un servicio de apoyo destinado a favorecer el proceso de inclusión educativa de los alumnos que presentan necesidades educativas especiales y prioritariamente aquellas asociadas con discapacidad y/o aptitudes sobresalientes, en las escuelas de educación inicial y básica de los diferentes niveles y modalidades educativas (SEP, 2006, p. 37).

Con este acercamiento se acuerda el contacto en las formas de comunicación e interacción con el profesorado en servicio, en construcción de una serie de responsabilidades que son parte de ese sentido de identidad y que corresponden a tomar parte y ser partícipe de algo. Este es el inicio del trayecto óptimo para transformar un camino hacia la liberación de la dependencia del estudiantado (Prieto, 2005), debido a que se definen las formas de participación y comunicación en la nueva normalidad para el grupo de estudiantes, lo implica el uso de la tecnología virtual: una forma diferente a la tradición previa y la primera que asume esta condición.

La importancia de empezar a generar una interpretación acerca de la realidad y la manera en que se vislumbra el acercamiento a la educación — con un enfoque de nueva normalidad - construye, desde la incertidumbre, la reflexión de que la función docente en tiempos de contingencia debe continuar. Es interesante la manera en la que el acercamiento asume condiciones hasta antes inexistentes en un inicio formativo, como lo es la dificultad para el estudiantado de insertarse en procesos que se construyen desde escenarios carentes de contacto físico, donde el análisis de la práctica -que se asume como una actividad en movilidad, de reflexión -, en relación con los acontecimientos que suceden entre profesorado y estudiantado (García et al., 2008), es parte activa en la construcción de la reflexión acerca de ser docente en tiempos complejos.

En la reflexión del estudiantado a través de su testimonio, su propia concepción de entender la experiencia en que se asume le dirige en este acercamiento a hacer presente el surgir de una categoría: la dificultad, debido a que vive situaciones inexploradas anteriormente en la propia formación tradicional de la que viene.

Hoy me he levantado muy temprano por los nervios, pues hoy vamos a tener una video llamada con el director y las maestras de USAER, donde nos asignarán la escuela con la que vamos a trabajar las jornadas de práctica. Cuando entré a la carrera, las prácticas se me hacían algo muy lejano, pero cuando menos lo esperé, ya nos estaban diciendo que nos asignarían una escuela (...) no me sentía lista para todo lo que venía, tenía bastante miedo de que las maestras que me tocaran fuesen muy estrictas o que no vieran potencial en mí. 
Durante la llamada, no sé cuántas veces escuché al director de USAER diciendo que nosotras debíamos ser las que buscáramos a las maestras (...) Luego le mandé un mensaje a la maestra, presentándome con ella y diciéndole que estaba a su disposición (...) yo ya no supe qué hacer exactamente (Estudiante 2, comunicación personal, 9 de octubre de 2020).

Entender que para quien estudia es un acercamiento que define su sentir y el ser docente debido a la experiencia en que está coexistiendo, imprime un importante sentido de la comprensión de la realidad que vive ahora (Vanegas y Fuantealba, 2019). Desde el testimonio del estudiantado novel, es claro que existe confusión y temor. Reflexionar sobre el cómo empiezan a vivir el proceso de iniciación a la práctica docente de forma diferente, permite identificar esos sentimientos, pero también se aprecia la necesidad y el deseo de la interacción con las otras personas, ya que esto forma parte de las funciones sociales como próximo profesorado.

En esta nueva realidad, reflexionar en la práctica educativa para entender lo que deben hacer para desarrollar el sentido docente dirige a planteamientos de diversas interrogantes como: ¿qué es posible hacer?, ¿qué objetivo se debe buscar al ir a la escuela de práctica?, ¿qué es factible hacer a través de los medios virtuales? y ¿qué método se adapta más a las respuestas educativas que se buscan? Interrogantes a las que deben encontrar respuestas que sumen a la forma de entender la docencia en tiempos complejos.

\subsection{Práctica en la nueva normalidad. El testimonio del estudiantado novel}

Ilustrar la manera en que, desde el estudiantado, se plasma la experiencia de vivir un programa de licenciatura en aislamiento, en contingencia, a la distancia y a través de escenarios virtuales en una nueva normalidad en tiempos complejos, invita a que se reflexione acerca de los pensamientos que construye la interacción con las situaciones propias a las que se enfrenta en la práctica escolar (Jiménez, 2020).

Como parte de estas reflexiones, las experiencias vividas son la base que apoya la idea del inicio de la formación del profesorado, las que aportan los elementos que construyen el sentido de identidad profesional en aislamiento.

Las actividades escolares en contingencia son asumidas a través de los roles estudiantiles que son semejantes a los de la escuela presencial, esto por ser parte de las relaciones que forman una pieza de la tradición educativa (Larrañaga, 2012). Como parte de los elementos de la cultura escolar, levantarse temprano y preparar los materiales para participar en la clase de forma remota son tareas de las costumbres que siguen presentes y que marcan fracciones de las acciones cotidianas de la escuela, para ajustarse a las diferentes formas que genera el acercamiento a lo desconocido, que se identifica como esa experiencia del profesorado titular en las escuelas de educación básica, para integrar aportes que den sentido al actuar en la práctica escolar.

Esta interacción que se da entre discentes noveles y profesorado en servicio, asume una ruta factible de desarrollar la práctica desde las interacciones en lejanía a través del discurso docente, lo que permite exponer la experiencia de vida de unas personas a otras, para construir con ellas el ejemplo escolar que se usa como base del sentido profesional para el estudiantado que carece de la experiencia real en escenarios físicos. Desde el discente, estas experiencias dan sentido a la edificación de los pensamientos a través de reconstruir las vivencias del profesorado.

Hoy me desperté a las 7:40 a.m. La verdad es que estaba muy cansada porque ayer me quedé un par de horas haciendo mi fondo para las clases virtuales, y ni siquiera siento que me haya quedado tan bonito como creí. Durante la llamada, las maestras nos comentaron a grandes rasgos la manera en que trabajan (...) Nos comentaron que ellas trabajan dándoles cuadernillos a los niños, y ellos mandan evidencia del trabajo realizado por semana o por día. Sin embargo, me pareció muy extraño que los niños no estén teniendo ninguna reunión con ellas, puesto que, si tampoco las toman con los maestros del aula, es probable que ya estén bastante atrasados respecto a sus compañeros (Estudiante 4, comunicación personal, 12 de octubre, 2020). 
A través de conocer la escuela desde la voz del personal docente y que el estudiantado profundice sobre su reflexión acerca de la educación, la docencia y la práctica escolar asumen una mirada sobre lo complejo que es la distancia y el hecho de interiorizar la idea particular sobre lo que es la propia formación (Castellanos y Yaya, 2013). Lo anterior, ante la carencia de experiencia en el escenario físico, construye en conjunto la continuidad de la reflexión escolar desde los saberes docentes y la propia vivencia previa en el trayecto escolar.

Es notoria la complejidad del aprendizaje en la práctica en estos escenarios virtuales, ya que es limitado el acercamiento con el alumnado de las escuelas de educación básica -ya sea por causas económicas, de accesibilidad o de recursos electrónicos (Montenegro y Fernández, 2017)-, derivado de las posibilidades de las familias para atender esta forma de acercamiento escolar. El profesorado en formación también percibe estas condiciones, lo que lo lleva a carecer de períodos de experiencia y suplir los momentos de aprendizaje profesional y dedicar el tiempo a la preparación de sus propias asignaturas. "Hoy no tuve sesiones, nadie se pudo conectar y me he centrado en mis tareas nada más" (Estudiante 5, comunicación personal 15 de octubre 2020).

La continuidad del programa de prácticas docentes a la distancia se presenta complejo, debido a las propias condiciones derivadas de las dificultades de conexión que facilitan la interacción y la participación con el estudiantado de educación básica, lo que limita que esta actividad sea constante y, por tanto, se ajuste a los momentos posibles, ya que los tiempos son limitados y están sujetos a los recursos de acceso de cada estudiante. Asimismo, son aprovechados en lo posible por parte del estudiantado de licenciatura, que los consideran elementos importantes para participar en la continuidad de la propuesta escolar.

Aunque es frustrante, esto carece de desmotivar al estudiantado en formación, que asume el acercamiento como parte del ser docente que están construyendo, y rescata el objetivo del mismo, el que se dirige a participar, conocer, teorizar, entender la interacción y relación de las partes educativas participantes en un proceso de enseñanza y aprendizaje. Desde la mirada de la persona que estudia, esta reflexión se aprecia en su testimonio:

Hoy a las 9:00 a.m. que comenzó la video llamada yo ya estaba muy nerviosa. Las maestras saludaban a los niños y yo sólo veía todo, luego me tocó. La verdad es que agradezco que los niños sean participativos y atendieron a las indicaciones. Posteriormente, la clase comenzó y fue muy difícil para mí. Me sorprendió mucho que, durante la llamada, los papás estuvieron al pendiente de todo. Sólo se conectaron ocho niños (de veintisiete), pero todos hicieron la misma actividad, la cual consistía en que la maestra lee un cuento corto, acompañado de señas e imágenes, y después se les planteaban oraciones a los niños, en la que ellos debían mostrar una palomita o una tacha en la pantalla, según si la oración era correcta o no.

Sin duda ha sido una experiencia muy agradable, a pesar de esas frustraciones y momentos de estrés, creo que el trabajo de las maestras de USAER ha sido bastante bueno. Claro que hay cosas por mejorar, para que todos los niños puedan seguir aprendiendo y no se queden atrás, pero el camino ha estado yendo bien (Estudiante 6, comunicación personal, 15 de octubre, 2020).

Es entonces que, desde la reflexión del testimonio del estudiantado, es posible interpretar que la práctica docente en tiempos complejos por la contingencia de salud es un proceso que también asume experiencias en su desarrollo. Lo anterior, aunque en la desigualdad social en la que el alumnado de todos los niveles participa, restringe el acceso a la cooperación en un trayecto formativo en el que todas las personas interactúen de forma activa. La práctica asume una reflexión con el compromiso del profesorado en formación, ya que lo invita a aprender desde la conjunción con el personal docente en servicio, para construir un pensamiento personal acerca de la escuela (Torres et al., 2020), la que, todavía en tiempos de pandemia, continúa siendo un espacio de interacciones en los procesos de formación social. 


\section{Conclusiones.}

Con la llegada de la contingencia de salud, el estudiantado novel de la escuela normal se ve exigido a dar una reinterpretación sobre las formas de llevar a cabo el acercamiento a la práctica, en la que el contacto es diferente a la idea tradicional y el trato cambia a interactuar con los recursos tecnológicos. Se tiene presente que estos acercamientos virtuales abren una nueva categoría a la que corresponde complejidad y la visión sobre la construcción de la propia formación profesional (Llerena, 2015). Es observable que el estudiantado, de forma autónoma y consciente, está en proceso de construcción de las capacidades para insertarse plenamente en los conocimientos sociales, ya que reflexiona en situaciones intrínsecas a los discentes en educación básica, y busca teorizar en la función didáctica del personal docente en educación inclusiva, donde el profesorado debe promover una intervención colaborativa. Lo anterior porque la configuración de la realidad —ante la emergencia de salud y en las nuevas dinámicas de interacción social y enfrentamiento a situaciones presentes como la falta de conexión del estudiantado, entrega de tareas, acciones de ayudantía, eventos, personas, entre otras - se desarrolla de manera cambiante y fluye según los contextos independientes de quien estudia, de la persona licenciada novel en formación, así como del profesorado en servicio que acompaña al estudiantado en preparación.

La interpretación de la práctica educativa en la que el estudiantado de la escuela normal reflexiona acerca de la dificultad de establecer procesos formativos con las partes participantes escolares, lo lleva —a través de la comunicación interpersonal con los maestros y las maestras titulares - a dirigirse a crear pensamientos particulares que serán generadores de procesos formativos (García et al., 2008), que se verán reflejados en su idea del ser docente.

A la distancia, aún en la dificultad manifiesta, la docencia novel en la práctica crea reflexiones positivas ante las situaciones de miedo, duda, aislamiento, desventaja, frustración y barreras en el acceso a la educación, lo que les permite reflexionar acerca de la función del profesorado en la institución escolar, al integrar pensamientos que forman parte de los trayectos de formación de la licenciatura y las propias percepciones culturales de la escuela. Exponerse a las necesidades de adaptación y generación de la realidad permite que el grupo de estudiantes genere una idea acerca de los procesos de educación que son llevados en la formación dada en las escuelas de enseñanza regular.

La tarea es difícil, la docencia es complicada y construir un sentido de identidad es un trabajo que se va haciendo de manera personal en la interacción con la tarea escolar, donde la persona discente novel debe ser parte activa en la búsqueda de acciones. Desde el texto es importante invitar al estudiantado a asumir esta experiencia en la contingencia de salud como una acción más que el personal docente debe tomar como parte de su tarea educativa, y que reconozca que la función docente carece de ser limitada por esta condición. Desde la reflexión académica, debe estar atento para atender y gestionar las acciones que lleven a experimentar, proponer, reflexionar, entender a su estudiantado de educación básica y reinterpretar que la escuela de acercamiento es eje rector de su significado como personas licenciadas en educación inclusiva para su futuro próximo profesional, aún en tiempos de amplia complejidad.

\section{REFERENCIAS}

Arriaga, H. (2015). El diagnóstico educativo, una importante herramienta para elevar la calidad de la educación en manos de los docentes. Atenas, 3(31), 63-74.

Castellanos, S. y Yaya, R. (2013). La reflexión docente y la construcción de conocimientos: una experiencia desde la práctica. Sinéctica, 41, 1-18.

Castro, V. (2015). Competencias administrativas y académicas en el profesorado de educación media superior. Revista mexicana de investigación educativa, 20(64), 263-294. 
Diario Oficial de la Federación [DOF]. (2020). Acuerdo número 02/03/20 por el que se suspenden las clases en las escuelas de educación preescolar, primaria, secundaria, normal y demás para la formación de maestros de educación básica del Sistema Educativo Nacional, así como aquellas de los tipos medio. https://www.dof.gob.mx/nota_detalle.php ?codigo $=5589479 \&$ fecha $=16 / 03 / 2020$

Escobar, M. (2005). Las competenicas laborales. ¿La estrategia laboral para la competitividad de las organizaciones?. Estudios Gerenciales, 21(96), 31-55.

García, B., Loredo, J. y Carranza, G. (2008). Análisis de la práctica educativa de los docentes: pensamiento, interacción y reflexión. Revista electrónica de investigación educativa, 10(spe), 1-15.

Jiménez, L. (2020, 1 de abril). Experiencias de enseñanza a distancia para llegar a todos los alumnos. Magisterio. https ://www.magisnet.com/2020/04/experiencias-de-ensenanza-a-distancia-ante-una-situacion-dificil/

Larrañaga, O. (2012). El modelo educativo tradicional frente a las nuevas estrategias de aprendizaje [tesis de maestría inédita]. Universidad Internacional de La Rioja.

Llerena, C. (2015). El proceso de formación profesional desde un punto de vista complejo e histórico - cultural. Revista Electrónica "Actualidades Investigativas en Educación", 15(3), 1-23.

Montenegro, S. y Fernández, F. (2017). La educación a distancia en entornos virtuales de enseñanza aprendizaje. Reflexiones didácticas. Atenas, 3(39).

Murillo, J. y Martínez, C. (2010). Investigación etnográfica. Métodos de invetigación educativa en educación especial. UAM.

Prieto, P. (2005). La participacipación de los estudaintes. ¿Un camino hacia su emancipación?. Theoria, 14(1), 27-36.

Rinesi, E., Singer, D. y Romé, N. (2020, 6 de julio). Infobae. https://bit.ly/3yNRw3v

Ruíz, C., Suarez, C., Meraz, M., Sánchez, R. y Chávez, C. (2010). Análisis de la práctica docente en el salón de clase desde la aplicación del instrumento de estrategias discursivas (ESTDI). Revista de la educación superior, 39(2), 7-17.

Secretaría de Educación Pública [SEP]. (2006). Orientaciones generales para el funcionamiento de los servicios de educación especial. Secretaría de Educación Pública.

Secretaría de Educación Pública [SEP]. (2018). Iniciación a la práctica docente en los servicios de educación especial Tercer Semestre. Dirección General de Educación Superior para Profesionales de la Educación.

SNTE. (2020). Sindicato Nacional de los Trabajadores de la Educación. https://snte.org.mx/razon-ser/

Téllez, L., Trejo, L. y Guzmán, E. (2014). Consejo de Transformación Educativa [Ponencia]. Primer Congreso Internacional de Transformación Educativa, México. https://www.transformacion-educativa.com/index.php/ articulos-sobre-educacion/74-la-practica-docente-entramados-y-repercusiones

Torres, M., Yépez, D. y Lara, A. (2020). La reflexión de la práctica docente. Revista Chakiñan de Ciencias Sociales y Humanidades, 10, 1-18.

Vanegas, C. y Fuantealba, A. (2019). Identidad profesional docente, reflexión y práctica pedagógica: Consideraciones claves para la formaicón de profesores. Perspectiva Educacional. Formación de Profesores, 58(1), 115-138.

Vergara, M. (2016). La práctica docente. Un estudio desde los significados. Revista CUMBRES, 2(1), 73-99.

\section{INFORMACIÓN ADICIONAL}

Cómo citar: Rangel Romero, J. C. (2022). El complejo inicio de la práctica docente en contingencia del estudiantado novel en educación inclusiva en tiempos de COVID-19. Revista Educación, 46(1). http://do i.org/10.15517/revedu.v46i1.44760 